\title{
Recurrent insulin-induced hypoglycemia induces AngII and COX2 leading to renal (pro)renin receptor expression and oxidative stress
}

\author{
Wael A. Alanazi ${ }^{1}$, Mohammad B. Uddin ${ }^{2}$, Selim Fakhruddin ${ }^{2}$, Keith E. Jackson ${ }^{2} *$ \\ ${ }^{1}$ Department of Pharmacology and Toxicology, College of Pharmacy, King Saud University, P.O. Box 2457, \\ Riyadh, 11451, Kingdom of Saudi Arabia. \\ ${ }^{2}$ Department of Basic Pharmaceutical Sciences, School of Pharmacy, University of Louisiana at Monroe, Monroe, LA 71201. USA \\ *Corresponding author E-mail: kjackson@ulm.edu
}

\begin{abstract}
Background: Recurrent insulin-induced hypoglycemia (RIIH) is an avoidable consequence in the therapeutic management of diabetes mellitus. RIIH has been implicated in causing hypertension through an increase in renal and systemic AngII production.

Objective: The present study was performed to assess the hypothesis that chronic insulin treatment enhances AngII and COX2 formation which in turn increases (pro) renin receptor (PRR) expression and NADPH oxidase-mediated oxidative stress, leading to renal and cardiac injury.

Methods: The present studies were conducted in Male Sprague Dawley rats treated with daily subcutaneous injections of $7 \mathrm{u} / \mathrm{kg}$ insulin or saline for 14 days. On the 14th day, surgery was performed for treatment infusion (captopril $12 \mathrm{mg} / \mathrm{kg}$, NS398 $0.3 \mathrm{mg} / \mathrm{kg}$ or vehicle), and renal interstitial fluid sample and urine collections for biomarker measurements. At the end of the experiments, kidneys and hearts were harvested to evaluate PRR and NOX2 (NADPH oxidase subunit) expression and oxidative stress.

Results: We found that RIIH enhanced AngII and COX2 activity, leading to renal PRR expression and NADPH oxidase-induced oxidative stress in the heart and kidney. 8-isoprostane was evaluated as a renal biomarker of oxidative stress, which was induced in insulin treated animals and modulated by captopril and NS398. In addition, there was a slight increase in NGAL, a urinary biomarker of acute kidney injury (AKI), in insulin treated animals when compared to control.

Conclusion: These results demonstrate that RIIH induces renal PRR expression and oxidative stress through increasing AngII and COX2 in the heart and kidney, leading to end-organ damage.
\end{abstract}

Keywords: Angiotensin II (AngII); Cyclooxygenase-2 (COX2); (Pro) Renin Receptor (PRR); Oxidative Stress; Recurring Insulin-Induced Hypoglycemia (RIIH).

\section{Introduction}

Free radicals are the byproducts of various enzymatic reactions in cellular metabolic processes. These byproducts have physiological and pathophysiological functions in several body systems (Bergendi et al. 1999, Dröge 2002). Free radical formation is associated with various cellular functions including signal transduction and gene transcription (Finkel 2011, Kunsch \& Medford 1999). In 1956, Denham Harman mentioned in his "free radical theory" that free radical formation occurs mainly during enzymatic reactions involving oxygen molecules and results in cellular damage at both the DNA and protein level (Harman, 1956). However, the imbalance in free radical production leads to disturbances in many biological processes, which promotes cellular dysfunction.

Reactive oxygen species such as superoxide (O2-), hydrogen peroxide, hydroxyl radical and peroxynitrite (ONOO-) have unpaired electrons in their outer orbit (Pavelescu 2015). The excess amount of these unpaired electrons destroys cellular lipids, proteins, and nucleic acids that have a role in different biological processes (Pruchniak et al. 2016). However, the overproduction of reactive oxygen species accelerates the incidence or complications of disor- ders for example diabetes mellitus, cardiovascular diseases, diabetic nephropathy, Alzheimer's disease, and Parkinson's disease (Popolo et al. 2013).

Several studies have shown the correlation between oxidative stress and the pathogenesis of both major types of diabetes as well as the development of diabetic complications (Yang et al. 2011, Giacco \& Brownlee 2010). In diabetic patients, free radicals are formed by different metabolic pathways such as glucose oxidation, degradation of glycated proteins, nonenzymatic glycation of proteins, and/or a decline of antioxidant defenses (Fiorentino et al. 2013). All these mechanisms participate in the elevation of oxidative stress, which in turn causes insulin resistance, and persist in the development of diabetic complications.

During the management of diabetes, hypoglycemia is the most common adverse complication which arises as one tries to obtain a state of euglycemia. In type1 diabetes (T1D), studies have shown that the progressive glycemic control caused up to 10 hypoglycemic episodes per week and produced mortality in $2-4 \%$ of patients with T1D (The DCCT Research Group 1993, The DCCT Research Group 1997, Cryer 2004). In addition, it has been documented that the frequency of hypoglycemic episodes in patients with type 2 diabetes (T2D) is more common in insulin and sulphonylurea-dependent T2D (Kalra et al. 2013, Shafiee et al. 2012). Severe hypoglyce- 
mia activates several physiological actions, causing autonomic activation which in turn induces release of adrenaline and other stress hormones. However, these stressors cause hemodynamic changes including an increase in the heart rate, stroke volume and systolic blood pressure as the body aims to maintain a continuous glucose supply to the brain (Wright \& Frier 2008). Diabetic patients with insulin therapy often have impaired awareness of hypoglycemia leading to disturbances in various organs such as the brain, heart, and kidney (Kalra et al. 2013). Previous studies have found a positive correlation between hypoglycemia and tachycardia, myocardial contractility, cytokine secretion and endothelial dysfunction (Fisher et al. 1990, Desouza et al. 2010). All of these are factors, which are known to contribute to the incidence and development of cardiovascular diseases.

Hypoglycemia which is associated with intensive insulin therapy or starvation has been indicated in the elevation of oxidative stress in different hypoglycemic models (Razavi Nematollahi et al. 2009, Bhardwaj et al. 1998). A previous study has found that the depletion of scavenging agents for instance glutathione S-transferase (GST), glutathione peroxidase (GPx), and glutathione reductase (GR) promote oxidative stress in the brain, liver and kidney during RIIH or starvation (Bhardwaj et al. 1998). On the other hand, NADPH oxidase was recently proposed as a source of hypoglycemia-induced oxidative stress during glucose reperfusion, which was blocked by apocynin treatment in in-vitro and in-vivo studies (Suh et al. 2007) Taken together, the literature does support activation of NADPH oxidase and/or inhibition of scavengers has a cause of oxidative stress development during hypoglycemic conditions.

The renin angiotensin aldosterone system (RAAS) has an essential role in maintaining blood pressure and body fluid hemostasis through interactions with various cellular pathways. Angiotensin II (AngII) is the major effector peptide in the RAAS, which is well known as a potent vasoconstrictor. As previously described, chronic AngII treatment caused an elevation in the mean arterial pressure, prostaglandin induction which lead to endothelial dysfunction and oxidative stress (King \& Fink 2006, Schrader et al. 2007, Hernández et al. 2002, Touyz \& Schiffrin 1999). We have previously shown that in addition to AngII mediated vasoconstriction, it also activates other pathways including enhancing carbon monoxide release (CO) by increasing HO-I (Heme oxygenase I), which leads to persistent hypertension (Quadri et al. 2013). Moreover, it has been documented that AngII plays a critical role in the development of hypertension and renal dysfunction through afferent arteriole vasoconstriction and sodium/water reabsorption (Baltatzi et al. 2011). In the renal vasculature, AngII via type 1 receptors (AT1) increases prostaglandin production by inducing PLA2 and COX2, which provides a counteracting mechanism to AngII-induced vasoconstriction (Hu et al. 2002). PGE2 and PGI2 modulate AngII-induced renal vasoconstriction, which would lead to a reduction in the renal blood flow (RBF) and glomerular filtration rate (GFR) (Imig 2006) Therefore, renal COX2-derived prostanoids, specifically PGE2 and PGI2 act as physiological buffers by maintaining normal blood pressure and renal excretory function.

In recent reports, AngII and COX2 have been targeted to investigate the mechanism for enhanced expression of (pro) renin receptor (PRR) during AngII-dependent hypertension. PRR has been identified recently as a receptor for renin and its precursor prorenin, where it induces local production of AngII (Nguyen et al. 2002). PRR expression has been shown to induce local RAS activation in the kidney, heart, brain and pancreas (Li et al. 2012). In the kidney, the PRR is localized in mesangial cells, intercalated type-A cells and podocytes (Nguyen et al. 1996, Ichihara et al. 2008, Advani et al. 2009). Intensive investigations have been undertaken to understand the role of AngII in the regulation of PRR expression in the kidney. In AngII-induced hypertension, studies have discovered that COX2-derived PGE2 play an essential role in enhancing PRR expression through binding to EP4 receptors in the renal medulla (Green et al. 2012, Wang et al. 2014, Yang 2015). However, crosstalk between renal medullary PRR and COX2-derived PGE2 has been documented during AngII-induced hypertension.
Several studies support the affirmation that AngII plays an essential role in superoxide production and nitric oxide (NO) reduction via induction of several pathophysiological pathways which in turn cause oxidative stress. In diabetic patients, RAAS activation promotes renal oxidative stress through NADPH oxidase-induced superoxide, and it also regulates nitric oxide synthase (NOS) in afferent arterioles through AT1 and AT2 receptors (Ratliff et al. 2010). Emerging evidence has found a circuitous relationship between cyclooxygenases (COX1 and COX2) and NADPH oxidase (Hernanz et al. 2010). Namely, ROS enhance cyclooxygenase production, which in turn induce ROS formation through prostanoids formation and other ROS generating pathways (Hernanz et al. 2010). In the current study, we hypothesize that RIIH augments PRR expression and oxidative stress through AngII and COX2 induction leading to cardiac and renal injury.

\section{Methods}

\subsection{Materials}

CMA 30 linear microdialysis probes were obtained from CMA/Microdialysis (Harvard Apparatus, Holliston, MA, USA). Humulin was purchased from Eli Lilly and Company (Indianapolis, IN, USA). 4-20\% gradient SDS-PAGE was obtained from Life Technologies Collaborations (Grand Island, NY, USA). Primary antibodies for PRR and NOX2 were purchased from Abcam (Cambridge, MA, USA). ELISA kits (8-iso-PGF2 $\alpha$ and NGAL), spin trapping reagents $(\mathrm{CMH}$ and $\mathrm{CPH})$ and diethyldithiocarbamic acid were obtained from Enzo Life Sciences (Farmingdale, NY, USA). Chemicals such as inactin (thiobutabarbital sodium), captopril, NS398 and 2-Methylbutane (isopentane) were purchased from Sigma-Aldrich (St. Louis, MO, USA). All other materials were obtained from Fisher Scientific (Houston, TX, USA).

\subsection{Animals}

Male Sprague-Dawley rats (200 - 250g, $n=20$, Harlan, Indianapolis, IN, USA) were housed in a controlled environment and had free access to food and water throughout the study. All experiments were approved by the University of Louisiana at Monroe Institutional Animal Care and Use Committee (IUCAC).

In the current study, we followed a previously described hypoglycemia model protocol, which was used to investigate the roles of hypoglycemia in neurological functions and hemodynamic changes (Paranjape \& Briski 2005, Quadri et al. 2014, Prathipati et al. 2015). Briefly, two groups of animals were treated subcutaneously with 7 units/kg of humulin insulin $(n=15)$ or saline $(n=5)$ for two weeks.

\subsection{Experimental procedure}

After two weeks of insulin treatment, rats were anaesthetized by inactin (thiobutabarbital sodium, $120 \mathrm{mg} / \mathrm{kg}$ ip). Surgical procedures were performed as previously described (Quadri et al. 2013, Alanazi et al. 2016). Briefly, the trachea was cannulated to allow an open airway throughout the experiment. The carotid artery, jugular vein and bladder were cannulated for hemodynamic evaluation, treatment infusion and urine sampling, respectively. After the cannulation procedures, rats were placed on their right side, and a small midsagittal incision was made to expose the left kidney. CMA 30 linear microdialysis probes were inserted into the renal cortex for renal interstitial sample collection.

\subsection{Treatment and samples collection}

After surgery, a $45 \mathrm{~min}$ recovery period was observed followed by a 4 hour control period in both normal and insulin treated groups. After the control period, the hypoglycemic animals were treated with a bolus dose of vehicle (7U+saline), captopril, $12 \mathrm{mg} / \mathrm{kg}$ (7U+captopril) or NS398, 0.3mg/kg (selective COX2 inhibitor) 
(7U+NS398). Throughout the experiments, urine and renal interstitial samples were collected and stored at $-80^{\circ} \mathrm{C}$ until analyzed for neutrophil gelatinase-associated lipocalin (NGAL) and 8-isoprostane (8-iso-PGF2 $\alpha$ ) analysis, respectively. At the end of the experiments, hearts and right kidneys were harvested and rapidly immersed in isopentane for snap freezing and stored at $-80^{\circ} \mathrm{C}$ until analyzed.

\subsection{Samples analysis}

\subsubsection{Western blot analysis}

As previously described, renal and cardiac homogenates were assayed for PRR and NOX2 proteins by western blotting (Gupta et al. 2012). Briefly, tissue lysates were prepared by using NP40 cell lysis buffer for protein extraction. Protein concentrations were determined via a bicinchoninic acid (BCA) protein assay kit. The obtained proteins were resolved by using 4-20\% gradient SDS-PAGE. After separation, proteins were transferred to a nitrocellulose membrane and blocked in 5\% fat-free milk in $0.05 \%$ Tween-20, $20 \mathrm{mM}$ phosphate buffered saline, pH 7.4 (PBST). The blots were incubated with anti-PRR or anti-NOX2 antibodies at $4{ }^{\circ} \mathrm{C}$ overnight and then washed with PBS and incubated with appropriate horseradish peroxide-conjugated secondary antibodies. Glyceraldehyde-3phosphate dehydrogenase (GAPDH) was used as a protein loading control.

\subsubsection{EPR spectrometer}

Electron Paramagnetic Resonance (EPR) spectrometer targets reactive oxygen species that have paramagnetic characteristics namely, O2- and ONOO-. The unpaired electrons of the O2- and ONOOabsorb the applied microwave radiation in a resonator cavity of EPR spectrometer within a static paramagnetic field that is produced by external magnets. A spectrum is generated due to the transition of the unpaired electrons to either parallel or anti-parallel direction to the magnetic field.

In the current study, O2- and ONOO- were measured in hearts and kidneys by using EPR spectrometer. CMH (1-hydroxy-3-methoxycarbonyl-2, 2, 5, 5-tetramethylpyrrolidine. $\mathrm{HCl}$ ) and $\mathrm{CPH}$ (1-hydroxy-3-carboxy-2, 2, 5, 5-tetramethylpyrrolidine. $\mathrm{HCl}$ ) were used as spin trapping reagents to trap O2- and ONOO- for measurement by EPR spectrometer. On the day of analysis, the stored hearts and kidneys were thawed and cut into $2 \mathrm{~mm}$ slices. The cardiac and renal pieces were incubated for 1 hour at $37^{\circ} \mathrm{C}$ in $0.5 \mathrm{ml}$ of DF/DETC/HEPES buffer containing $5 \mathrm{mM}$ of $\mathrm{CMH}$ or $\mathrm{CPH}$ (Dikalov et al. 2007). After incubation, a micropipette was filled with the sample solution to be ready for EPR analysis. EPR spectrometer was adjusted to the following settings: field sweep, 80G; microwave frequency, $9.64 \mathrm{GHz}$; microwave power, $1.34 \mathrm{~mW}$; modulation amplitude, 5G; conversion time, $327.68 \mathrm{~ms}$; time constant, $10.24 \mathrm{~ms}$; 512 points resolution; and receiver gain, $1 \times 10^{4}$.

\subsubsection{8-isoprostane analysis}

8 -iso- $\mathrm{PGF}_{2 \alpha}$ or 8 -isoprostane is known as a biomarker of oxidative stress (Cracowski et al. 2002). The collected renal interstitial samples were analyzed for the presence of 8-isoprostane to confirm the induction of oxidative stress during RIIH. The collected renal interstitial samples were analyzed by commercially available ELISA kits.

\subsubsection{Neutrophil gelatinase-associated lipocalin (NGAL) analy- sis}

In the present study, the urinary NGAL was measured as a biomarker of acute kidney injury (AKI) (Devarajan 2010). Urine samples were analyzed by commercially available ELISA kits.

\subsection{Statistics}

Data were expressed as mean $\pm \mathrm{SE}$ and analyzed by one-way analysis of variance (ANOVA) followed by Tukey-Kramer multiple comparison test when appropriate (INSTAT 3$)$. (P < 0.05) was considered significant.

\section{Results}

\subsection{PRR expression}

Western blot analysis of PRR showed a significant increase in PRR protein abundance in the kidney during RIIH (7U+vehicle) as compared with control. Captopril (7U+captopril) and NS398 (7U+NS398) treatment reduced RIIH-induced renal PRR protein (Fig.1). In the heart, RIIH enhanced the abundance of PRR protein but captopril and NS398 administration did not return PRR protein to control levels (Fig.2).

PRR GAPDH
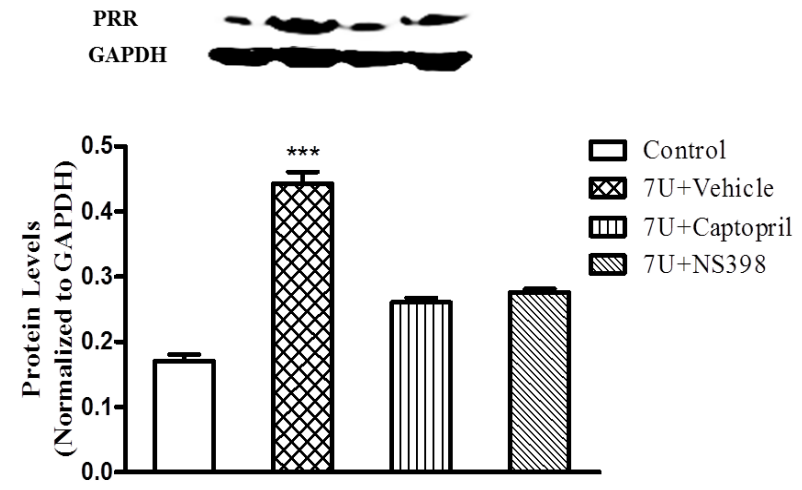

Fig. 1: PRR Expression in the Kidney. A Significant Increase in PRR Expression was Observed during RIIH as Compared with Control $(* * * \mathrm{P}<0.001)$. Captopril and NS398 Significantly Decreased PRR Expression during RIIH. Values are expressed as Mean \pm SE.

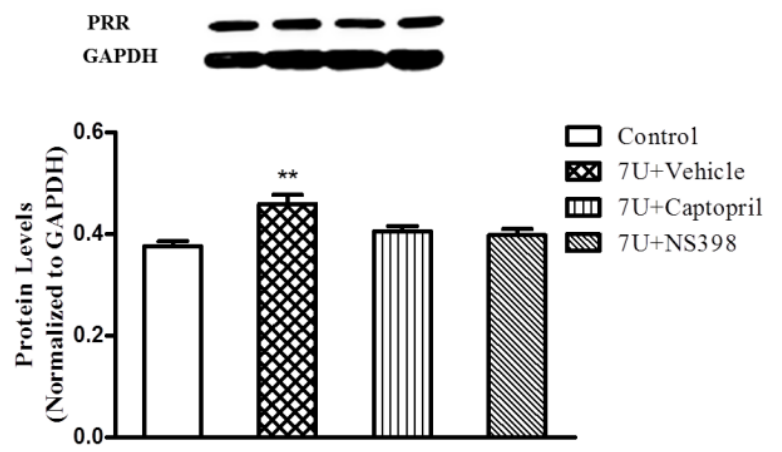

Fig. 2: PRR Expression in the Heart. A Significant Increase in PRR Expression was Observed during RIIH as Compared with Control $(* * \mathrm{P}<0.01)$. Captopril and NS398 Didn't Change PRR Expression Significantly during $\mathrm{RIIH}$. Values are expressed as Mean $\pm \mathrm{SE}$.

\subsection{Superoxide and peroxynitrite measurement in the heart}

Two weeks of $7 \mathrm{U} / \mathrm{Kg}$ insulin treatment induced ROS (O2- and ONOO-) formation significantly in the heart when compared to saline treated animals (Fig.3) (Fig.4). The incubated cardiac tissues with $\mathrm{CMH}$ and $\mathrm{CPH}$ showed a massive induction of $\mathrm{O} 2-$ and ONOO- in 7U+vehicle treated animals as compared to the control. Captopril treatment decreased O2- and ONOO-formation in the $7 \mathrm{U}+$ captopril group and returned them to the normal levels when compared to 7U+vehicle and control groups. NS398 treatment reduced RIIH-induced O2- and ONOO- significantly in 7U+NS398 group as compared to 7U+vehicle group (Fig.3) (Fig.4). 


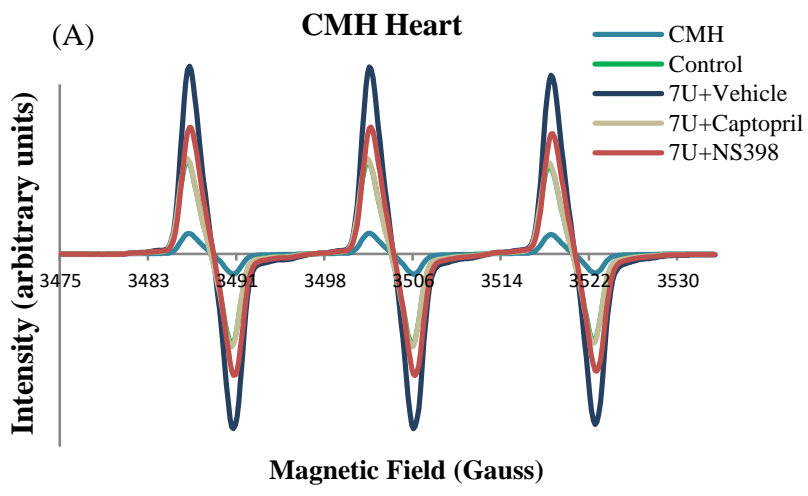

(B)

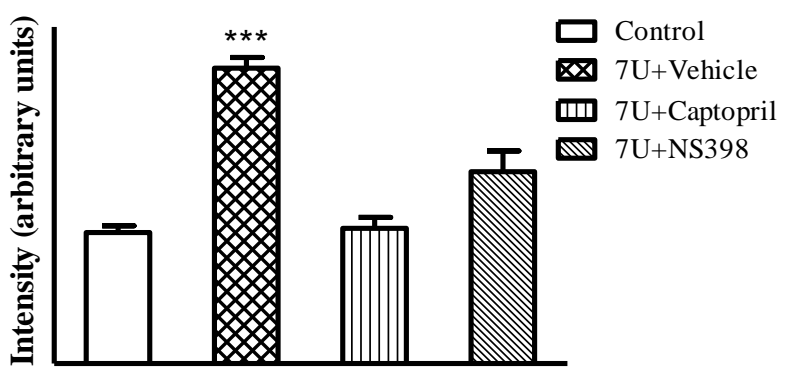

Fig. 3: Superoxide Measurement in the Heart. (A) Spectra were obtained from EPR Spectrometer where Cardiac Samples were incubated with CMH. (B) Bar Graphs were derived from the Peaks of EPR Spectra to Compare Superoxide Levels in the Hearts of All Groups. (*** P <0.001) Values are expressed as Mean \pm SE.

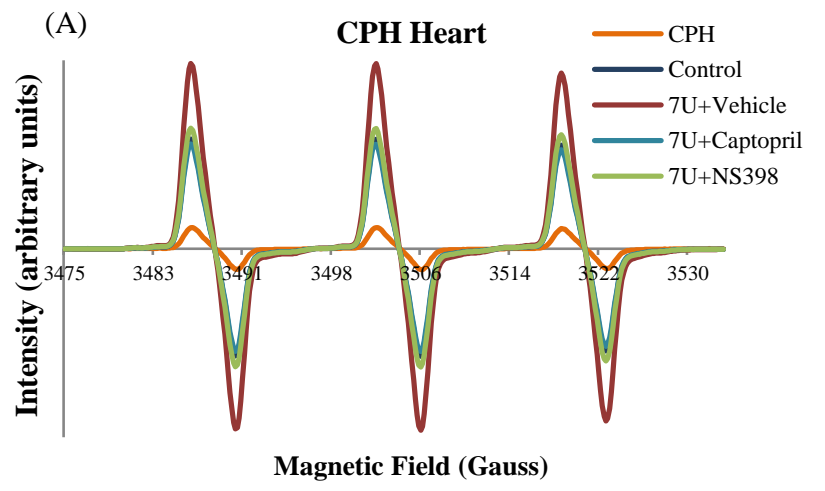

(B)

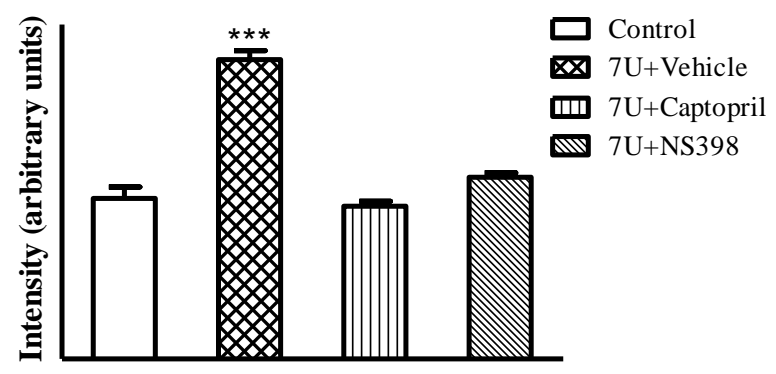

Fig. 4: Peroxynitrite Measurement in the Heart. (A) Spectra were obtained from EPR Spectrometer Where Cardiac Samples were incubated with $\mathrm{CPH}$ (B) Bar Graphs were derived from the Peaks of EPR Spectra to Compare Peroxynitrite Levels in the Hearts of All Groups. (***P <0.001) Values are expressed as Mean \pm SE.

\subsection{Superoxide and peroxynitrite measurement in the kidney}

O2- and ONOO- levels were significantly elevated in the kidney during two weeks of $7 \mathrm{U}$ insulin treatment as compared to saline treated animals (Fig.5) (Fig.6). Incubation of renal slices with CMH and $\mathrm{CPH}$ allowed EPR spectrometer to record significant increases in $\mathrm{O} 2$ - and $\mathrm{ONOO}-$ levels in $7 \mathrm{U}$ insulin treated rats when compared to the control group. In 7U+captopril group, captopril blocked O2and ONOO- formation and returned them to the normal levels during RIIH as compared to 7U+vehicle and control groups. NS398 reduced O2- and ONOO- induction in 7U+NS398 treated animals when compared to $7 \mathrm{U}+$ vehicle group (Fig.5) (Fig.6).

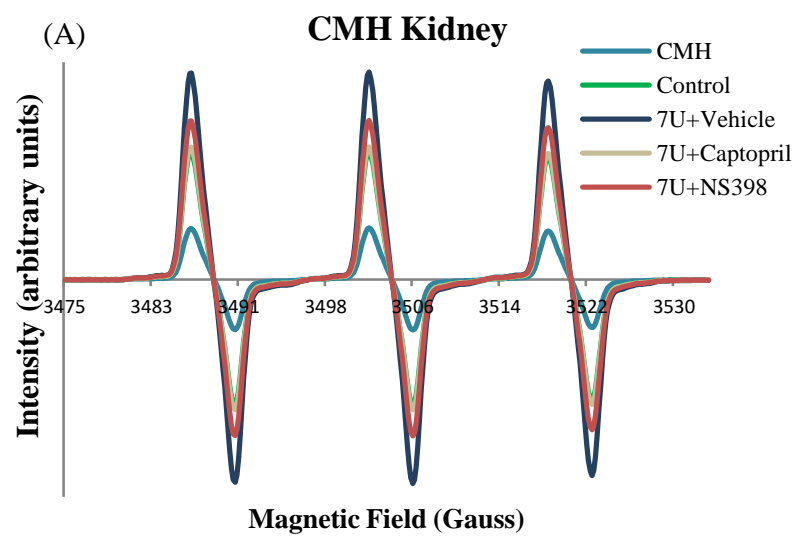

(B)

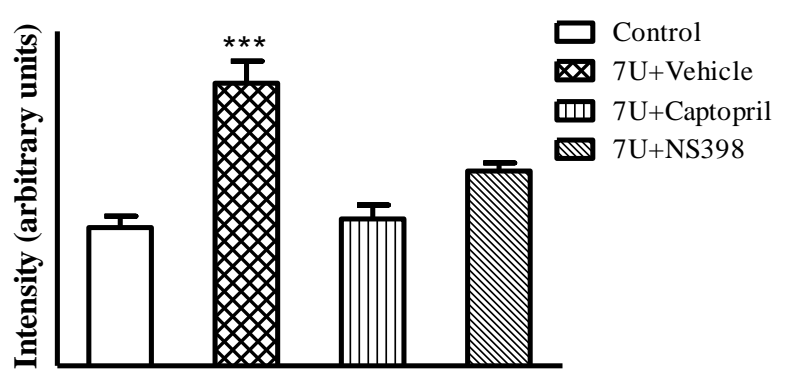

Fig. 5: Superoxide Measurement in the Kidney. (A) Spectra were obtained from EPR Spectrometer Where Renal Samples Were Incubated with CMH. (B) Bar Graphs were derived from the Peaks of EPR Spectra to Compare Superoxide Levels in the Kidneys of All Groups. (***P $<0.001)$ Values are expressed as Mean $\pm \mathrm{SE}$.

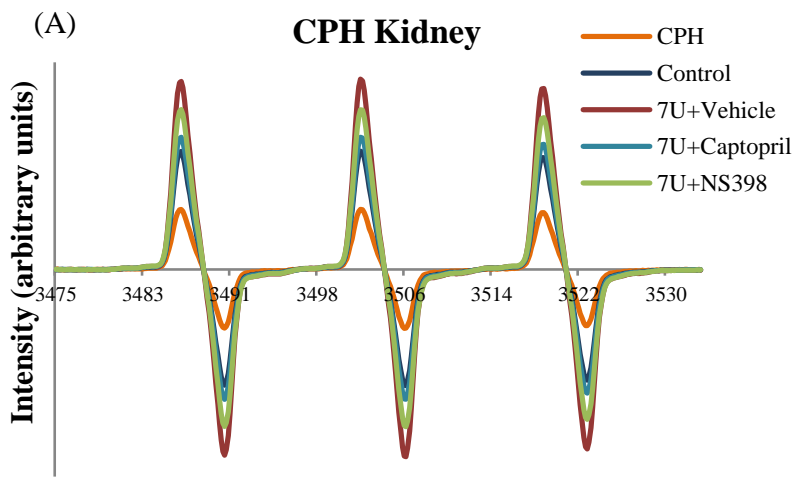

Magnetic Field (Gauss) 
(B)

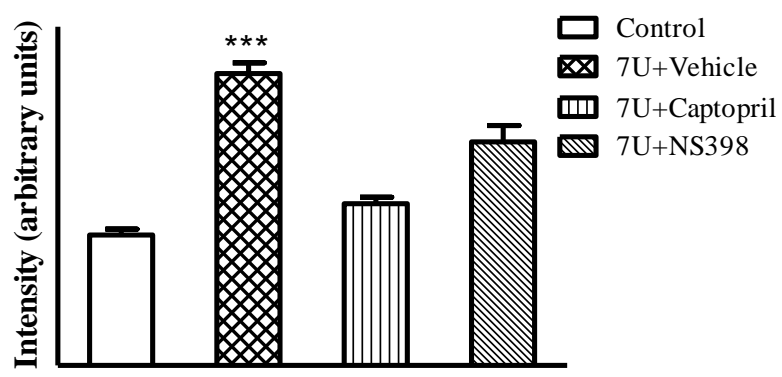

Fig. 6: Peroxynitrite Measurement in the Kideny. (A) Spectra were obtained from EPR Spectrometer where Renal Samples Were Incubated With CPH. (B) Bar Graphs were derived from The Peaks of EPR Spectra to Compare Peroxynitrite Levels in the Kidneys of All Groups. (*** $\mathrm{P}<0.001)$ Values are expressed as Mean $\pm \mathrm{SE}$.

\subsection{NOX2 expression}

Western blot analysis of NOX2 showed a significant increase in NOX2 protein abundance in the kidney and heart during RIIH (7U+vehicle) as compared with control. Captopril (7U+captopril) and NS398 (7U+NS398) treatment reduced RIIH-induced NOX2 protein in the kidney and heart (Fig.7) (Fig.8).
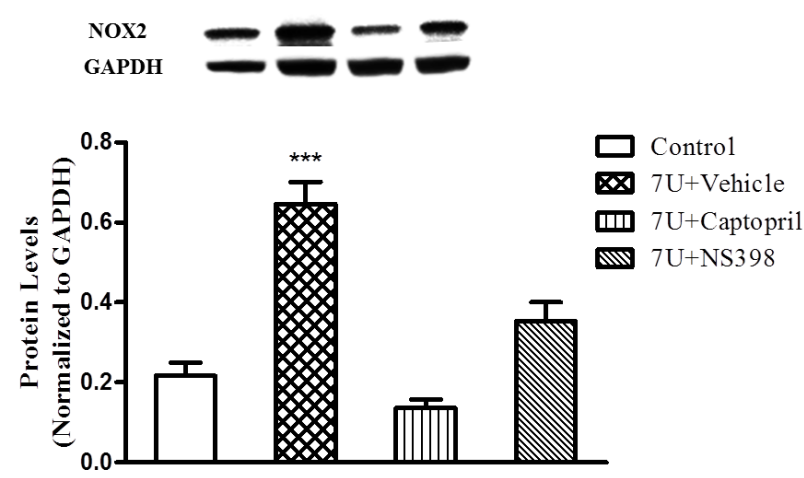

Fig. 7: NOX2 Expression in the Kidney. A Significant Increase in NOX2 Expression was Observed during RIIH as Compared with Control $(* * * \mathrm{P}<0.001)$. Captopril and NS398 Significantly Decreased NOX2 Expression during RIIH. Values are expressed as Mean $\pm \mathrm{SE}$.
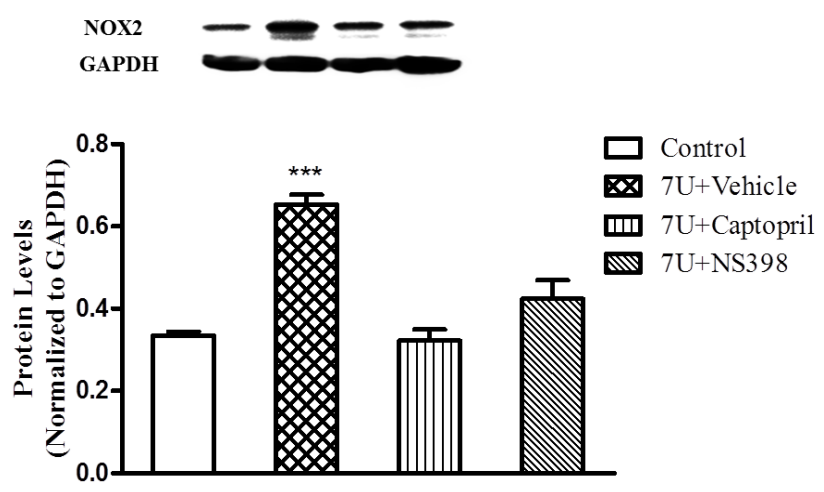

Fig. 8: NOX2 Expression in the Heart. A Significant Increase in NOX2 Expression was Observed during RIIH as Compared with Control $(* * * \mathrm{P}<0.001)$. Captopril and NS398 Significantly Decreased NOX2 Expression during RIIH. Values are expressed as Mean $\pm \mathrm{SE}$.

\subsection{8-isoprostane measurement in the renal interstitial fluid}

In $7 \mathrm{U}+$ vehicle treated group, 8 -isoprostane was elevated significantly $(834.55 \pm 28.42 \mathrm{pg} / \mathrm{ml})$ as compared with the control group
(559.92 $\pm 30.46 \mathrm{pg} / \mathrm{ml})$. Captopril and NS398 blocked the RIIH-induced oxidative stress in $7 \mathrm{U}+$ captopril $(530.34 \pm 16.83 \mathrm{pg} / \mathrm{ml})$ and $7 \mathrm{U}+\mathrm{NS} 398(531.53 \pm 24.01 \mathrm{pg} / \mathrm{ml})$ treated groups respectively when compared to $7 \mathrm{U}+\mathrm{vehicle}$ and control groups (Fig.9).

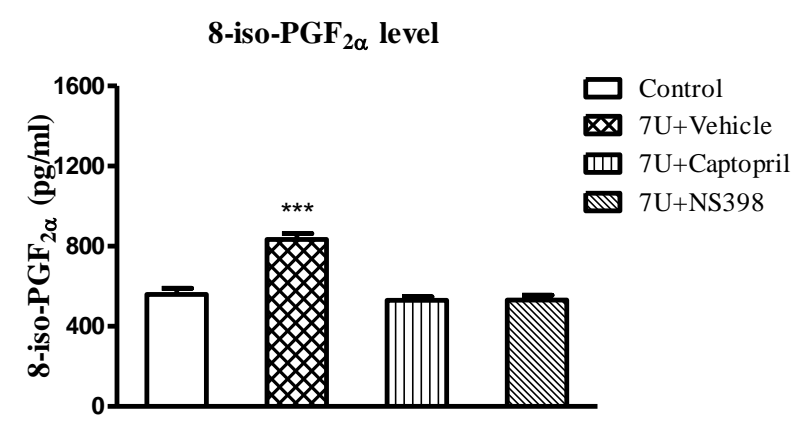

Fig. 9: 8-Isoprostane Levels in the Renal Interstitial Fluid. 8-Isoprostane Was Measured In Insulin with Vehicle (7U+Vehicle), Insulin with Captopril (7U+Captopril), Insulin with NS398 (7U+NS398) and Control Treated Rats. (*** $\mathrm{P}<0.001)$ Values are expressed as Mean $\pm \mathrm{SE}$.

\subsection{NGAL measurement in the urine}

The urinary NGAL level in 7U+vehicle treated group was slightly increased during the hypoglycemic condition $(2.77 \pm 0.31 \mu \mathrm{g} / \mathrm{ml})$ as compared to the control group $(2.36 \pm 0.27 \mu \mathrm{g} / \mathrm{ml})$. Captopril and NS398 treatment showed no significant change in NGAL levels during RIIH (Fig.10).

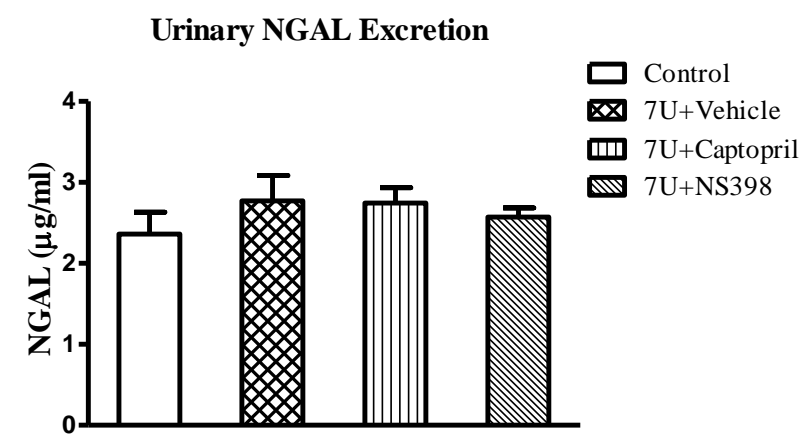

Fig. 10: Urinary NGAL Measurement. NGAL was Measured in Insulin with Vehicle (7U+Vehicle), Insulin with Captopril (7U+Captopril), Insulin with NS398 (7U+NS398) and Control Treated Rats.

\section{Discussion}

The present study investigates the role of AngII and COX2 during RIIH in inducing PRR expression and development of oxidative stress, which promotes organ damage. The current results discovered for the first time that RIIH increases PRR expression in the renal tissues. Also, our results found that the inhibition of AngII and COX2 blocked RIIH-induced PRR expression, which confirmed the essential roles of AngII and COX2 in increasing renal PRR expression during RIIH. Previous studies documented that COX2/PGE2/EP4 pathway is responsible for high PRR expression in the renal medulla during AngII-induced hypertension (Green et al. 2012, Wang et al. 2014, Yang 2015). However, in the current study, RIIH induced AngII which might activate the COX2/PGE2/EP4 pathway and lead to an increase in expression of PRR in the kidney. In the heart, we found that RIIH also increased PRR expression but AngII and COX2 inhibition showed no significant changes in PRR expression during RIIH. Hence, our results revealed that RIIH activates AngII/COX2/EP4 pathway, leading to increasing the local RAS activity in the kidney through inducing PRR expression. Further investigation is required to determine the mechanism for increased PRR expression in the heart. 
Previous reports have demonstrated that hypoglycemia increases production of reactive oxygen species, however the underlining mechanism is still unclear (Haces et al. 2010, McGowan et al. 2006, Pàramo et al. 2010). Our previous results have shown that AngII induced hypertension during RIIH promotes an increase in $\mathrm{CO}$ levels through the inducible HO enzyme, HO-1 (Quadri et al. 2014). Through daily measurements of renal interstitial ATP and AngII, we found that RIIH augmented ATP and AngII leading to attenuation in tubuloglomerular feedback (TGF) which might increase oxidative stress in kidneys (Prathipati et al. 2015). However, in the current study, we found that AngII inhibition reduced RIIH-induced reactive oxygen species formation (O2- and ONOO-) in renal and cardiac tissues. These results validated that RIIH-induced AngII is involved in causing oxidative stress. In recent reports of our lab, RIIH was shown to activate renal COX2-derived prostanoids, which are known to enhance superoxide formation (Wong et al. 2010, Tian et al. 2012, Alanazi et al. 2016 in press). Hence, we targeted COX2 to investigate its role in the development of renal and cardiac oxidative stress during RIIH. As anticipated, COX2 inhibition showed a reduction in O2- and ONOO- in the hypoglycemic animals, thus demonstrating that COX2 plays a role in RIIH-induced oxidative stress. Taken together, RIIH augments reactive oxygen species formation via activation of AngII and COX2-derived prostanoids. In addition, RIIH might also enhance superoxide formation via PRR activation, which was recently implicated in arterial smooth muscle cell induction of superoxide release (Liu et al. 2014).

For further investigation, renal interstitial samples were evaluated for the presence of oxidative stress through measurement of 8-isoprostane, which is a biomarker of oxidative stress. Renal 8-isoprostane levels were elevated during RIIH demonstrating the presence of oxidative stress in the kidney. AngII and COX2 inhibition attenuated the elevation in renal 8-isoprostane, thus demonstrating the underlining mechanism for oxidative stress formation in the kidney. Moreover, RIIH-induced AKI was evaluated through measurement of urinary NGAL, which showed a slight increase in NGAL levels that might indicate mild AKI. Hence, these results suggest that long term hypoglycemic episodes might be associated with progression to chronic kidney diseases (CKD) in insulin-dependent diabetes mellitus.

AngII has been implicated in causing oxidative stress in numerous diseases including hypertension and diabetes via enhanced superoxide formation mainly through activation of NADPH oxidase (Dikalov \& Nazarewicz 2013). AngII-bound AT1 receptors mediate the translocation of cytoplasmic subunits of NADPH oxidase to attach to the cell membrane subunits for activation (Wei et al. 2006). ACE inhibition (captopril) and AT1 receptor blockade (losartan) modulated AngII-induced superoxide formation through reduced NADPH oxidase activation and an increase in NO bioavailablity (Rajagopalan et al. 1996, van der Giet et al. 2002, Deng et al. 2009). It is suggested that AngII impairs production of endothelial NO through a reduction in eNOS activity, during chronic disease states including diabetes, diabetic nephropathy and hypertension (Zhang et al. 2003, Toda et al. 2007).

The literature supports the potential for crosstalk between COX2 and NADPH oxidase (Cheng et al. 2013, Hernanz et al. 2014). A previous report showed that COX2 plays a significant role in AngIIinduced NADPH oxidase-derived O2-production (Wu et al. 2005). Rofecoxib (Selective COX2 inhibitors) blocked superoxide formation and reduced cardiac hypertrophy, which provided antioxidative properties during enhanced AngII activity (Wu et al. 2005). Collectively, these results demonstrate that AngII induces oxidative stress either directly through activation of reactive oxygen species sources or via an enhancement in COX2 activity. This synergistic action of AngII might be an amplifying mechanism, which leads to organ damage that is commonly associated with serious disease stages like hypertension. However, further investigation is required to clarify the particular role of each prostanoid in the activation of AngII/COX2/NADPH oxidase pathway that is correlated with oxidative stress during hypoglycemic conditions.

\section{Conclusion}

The current results demonstrated that AngII and COX2 play an essential role in promoting renal PRR expression and oxidative stress during RIIH (Fig.11). Hence, AngII blockade and COX2 inhibition maybe critical therapeutic targets for the prevention of RIIH-mediated renal and cardiovascular injury.

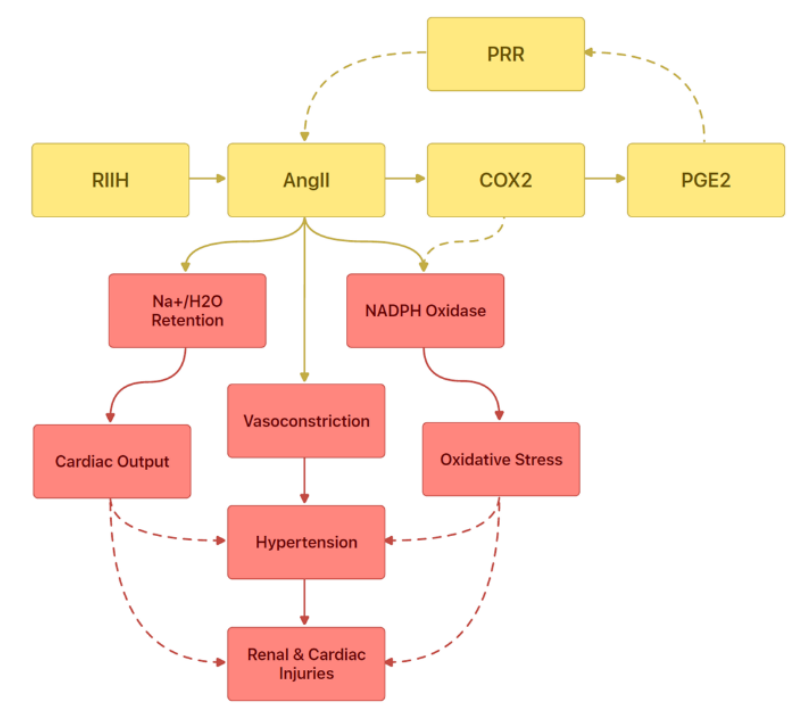

Fig. 11: Summary of RIIH-Induced Renal PRR Expression and Oxidative Stress.

\section{Acknowledgment}

This work was supported by a NIH-LBRN grant and a Saudi Arabian Cultural Mission (SACM) fellowship. We would like to sincerely thank Dr. Sharon Meyer for her assistance with the EPR experiments and Dr. Yong-Yu Liu for his contribution in the review process.

\section{References}

[1] Advani A, Kelly DJ, Cox AJ, White KE, Advani SL, Thai K, Connelly KA, Yuen D, Trogadis J, Herzenberg AM, Kuliszewski MA, Leong-Poi H \& Gilbert RE (2009) The (Pro)renin receptor: site specific and functional linkage to the vacuolar H+-ATPase in the kidney. Hypertension. 54, 261-269. https://doi.org/10.1161/HYPERTENSIONAHA.109.128645.

[2] Alanazi W, Fakhruddin S \& Jackson KE (2016) Microdialysis Sampling of Renal Interstitial Fluid in Acute Studies. International Journal of Biology 8, 69-79. https://doi.org/10.5539/ijb.v8n1p69.

[3] Baltatzi M, Savopoulos C \& Hatzitolios A (2011) Role of angiotensin converting enzyme inhibitors and angiotensin receptor blockers in hypertension of chronic kidney disease and renoprotection. Study results. Hippokratia 15, 27-32.

[4] Bergendi L, Benes L, Duracková Z \& Ferencik M (1999) Chemistry, physiology and pathology of free radicals. Life Sci. 65, 1865-74. https://doi.org/10.1016/S0024-3205(99)00439-7.

[5] Bhardwaj SK, Sharma ML, Gulati G, Chhabra A, Kaushik R, Sharma P \& Kaur G (1998) Effect of starvation and insulin-induced hypoglycemia on oxidative stress scavenger system and electron transport chain complexes from rat brain, liver, and kidney. Mol Chem Neuropathol. 34, 157-68. https://doi.org/10.1007/BF02815077.

[6] Cheng SE, Lee IT, Lin CC, Wu WL, Hsiao LD \& Yang CM (2013) ATP mediates NADPH oxidase/ROS generation and COX-2/PGE2 expression in A549 cells: role of P2 receptor-dependent STAT3 activation. PLoS One. 8, e54125. https://doi.org/10.1371/journal.pone.0054125.

[7] Cracowski JL, Durand T \& Bessard G (2002) Isoprostanes as a biomarker of lipid peroxidation in humans: physiology, pharmacology and clinical implications. Trends Pharmacol Sci. 23, 360-6. https://doi.org/10.1016/S0165-6147(02)02053-9.

[8] Cryer PE (2004) Current concepts: Diverse causes of hypoglycemiaassociated autonomic failure in diabetes. N Engl J Med. 350, 2272 9. https://doi.org/10.1056/NEJMra031354. 
[9] Deng A, Tang T, Singh P, Wang C, Satriano J, Thomson SC \& Blantz RC (2009) Regulation of oxygen utilization by angiotensin II in chronic kidney disease. Kidney Int. 75, 197-204. https://doi.org/10.1038/ki.2008.481.

[10] Desouza CV, Bolli GB \& Fonseca V (2010) Hypoglycemia, diabetes, and cardiovascular events. Diabetes Care. 33, 1389-94. https://doi.org/10.2337/dc09-2082.

[11] Devarajan P (2010) Neutrophil gelatinase-associated lipocalin: a promising biomarker for human acute kidney injury. Biomark Med. 4, 265-280. https://doi.org/10.2217/bmm.10.12.

[12] Dikalov S, Griendling KK \& Harrison DG (2007) Measurement of reactive oxygen species in cardiovascular studies. Hypertension. 49 717-727. https://doi.org/10.1161/01.HYP.0000258594.87211.6b.

[13] Dikalov SI \& Nazarewicz RR (2013) Angiotensin II-induced production of mitochondrial reactive oxygen species: potential mechanisms and relevance for cardiovascular disease. Antioxid Redox Signal. 19, 1085-94. https://doi.org/10.1089/ars.2012.4604.

[14] Dröge W (2002) Free radicals in the physiological control of cell function. Physiol Rev. 82, 47-95. https://doi.org/10.1152/physrev.00018.2001.

[15] Finkel T (2011) Signal transduction by reactive oxygen species. J Cell Biol. 194, 7-15. https://doi.org/10.1083/jcb.201102095.

[16] Fiorentino TV, Prioletta A, Zuo P \& Folli F (2013) Hyperglycemiainduced oxidative stress and its role in diabetes mellitus related cardiovascular diseases. Curr Pharm Des. 19, 5695-703. https://doi.org/10.2174/1381612811319320005.

[17] Fisher BM, Gillen G, Hepburn DA, Dargie HJ \& Frier BM (1990) Cardiac responses to acute insulin-induced hypoglycemia in humans. Am J Physiol. 258, H1775-9.

[18] Giacco F \& Brownlee M (2010) Oxidative stress and diabetic complications. Circ Res. 107, 1058-1070. http://dx.doi.org/10.1161/CIRCRESAHA.110.223545.

[19] Green T, Gonzalez AA, Mitchell KD \& Navar LG (2012) The complex interplay between cyclooxygenase- 2 and angiotensin II in regulating kidney function. Curr Opin Nephrol Hypertens. 21, 7-14. https://doi.org/10.1097/MNH.0b013e32834d9d75.

[20] Gupta V, Bhinge KN, Hosain SB, Xiong K, Gu X, Shi R, Ho MY, Khoo KH, Li SC, Li YT, Ambudkar SV, Jazwinski SM, Liu YY (2012) Ceramide glycosylation by glucosylceramide synthase selectively maintains the properties of breast cancer stem cells. J Biol Chem. 287, 37195-205. https://doi.org/10.1074/jbc.M112.396390.

[21] Haces ML, Montiel T \& Massieu L (2010) Selective vulnerability of brain regions to oxidative stress in a non-coma model of insulin-induced hypoglycemia. Neuroscience 165, 28-38. https://doi.org/10.1016/j.neuroscience.2009.10.003.

[22] Harman D (1956) Aging: a theory based on free radical and radiation $\begin{array}{llll}\text { chemistry. } & \text { J } & \text { Gerontol. }\end{array}$ https://doi.org/10.1093/geronj/11.3.298.

[23] Hernández J, Astudillo H \& Escalante B (2002) Angiotensin II stimulates cyclooxygenase- 2 mRNA expression in renal tissue from rats with kidney failure. Am J Physiol Renal Physiol. 282, F592-8. https://doi.org/10.1152/ajprenal.00194.2001.

[24] Hernanz R, Briones AM, Salaices M \& Alonso MJ (2014) new roles for old pathways? A circuitous relationship between reactive oxygen species and cyclo-oxygenase in hypertension. Clin Sci (Lond). 126, 111-21. https://doi.org/10.1042/CS20120651.

[25] Hu ZW, Kerb R, Shi XY, Wei-Lavery T \& Hoffman BB (2002) Angiotensin II increases expression of cyclooxygenase-2: implications for the function of vascular smooth muscle cells. J Pharmacol Exp Ther. 303, 563-573. https://doi.org/10.1124/jpet.102.037705.

[26] Ichihara A, Sakoda M, Kurauchi-Mito A, Kaneshiro Y \& Itoh H (2008) Involvement of (pro)renin receptor in the glomerular filtration barrier. J Mol Med. 86, 629-635. https://doi.org/10.1007/s00109008-0327-1.

[27] Imig JD (2006) Eicosanoids and renal vascular function in diseases Clin Sci. 111, 21-34. https://doi.org/10.1042/CS20050251.

[28] Kalra S, Mukherjee JJ, Venkataraman S, Bantwal G, Shaikh S, Saboo B, Das AK \& Ramachandran A (2013) Hypoglycemia: The neglected complication. Indian J Endocrinol Metab. 17, 819-34. https://doi.org/10.4103/2230-8210.117219.

[29] King AJ \& Fink GD (2006) Chronic low-dose angiotensin II infusion increases venomotor tone by neurogenic mechanisms. Hypertension. 48, 927-33. https://doi.org/10.1161/01.HYP.0000243799.84573.f8.

[30] Kunsch C \& Medford RM (1999) Oxidative stress as a regulator of gene expression in the vasculature. Circ Res. 85, 753-66. https://doi.org/10.1161/01.RES.85.8.753.

[31] Li W, Peng H, Cao T, Sato R, McDaniels SJ, Kobori H, Navar LG \& Feng Y (2012) Brain-targeted (pro)renin receptor knockdown attenuates angiotensin II-dependent hypertension. Hypertension. 59,
1188-94.

https://doi.org/10.1161/HYPERTENSIONAHA.111.190108.

[32] Liu FY, Liu XY, Zhang LJ, Cheng YP, Jiang YN (2014) Binding of prorenin to (pro)renin receptor induces the proliferation of human umbilical artery smooth muscle cells via ROS generation and ERK1/2 activation. J Renin Angiotensin Aldosterone Syst. 15, 99108. https://doi.org/10.1177/1470320314525215.

[33] McGowan JE, Chen L, Gao D, Trush M \& Wei C (2006) Increased mitochondrial reactive oxygen species production in newborn brain during hypoglycemia. Neurosci Lett. 399, 111-114 https://doi.org/10.1016/j.neulet.2006.01.034.

[34] Nguyen G, Delarue F, Berrou J, Rondeau E \& Sraer JD (1996) Specific receptor binding of renin on human mesangial cells in culture increases plasminogen activator inhibitor-1 antigen. Kidney Int. 50, 1897-1903. https://doi.org/10.1038/ki.1996.511.

[35] Nguyen G, Delarue F, Burckle C, Bouzhir L, Giller T \& Sraer JD (2002) Pivotal role of the renin/prorenin receptor in angiotensin II production and cellular responses to renin. J Clin Invest. 109, 141727. https://doi.org/10.1172/JCI0214276.

[36] Pàramo B, Hernandez-Fonseca K, Estrada-Sanchez AM, Jimenez N, Hernandez-Cruz A \& Massieu L (2010) Pathways involved in the generation of reactive oxygen and nitrogen species during glucose deprivation and its role on the death of cultured hippocampal neurons. Neuroscience 167, 1057-1069. https://doi.org/10.1016/j.neuroscience.2010.02.074

[37] Paranjape SA \& Briski KP (2005) recurrent insulin-induced hypoglycemia causes site-specific patterns of habituation or amplification of CNS neuronal genomic activation. Neuroscience 130, 957-70. https://doi.org/10.1016/j.neuroscience.2004.09.030.

[38] Pavelescu LA (2015) on reactive oxygen species measurement in living systems. J Med Life. 8, 38-42.

[39] Popolo A, Autore G, Pinto a \& Marzocco S (2013) Oxidative stress in patients with cardiovascular disease and chronic renal failure. Free Radic

Res. 47 , 346-56. https://doi.org/10.3109/10715762.2013.779373.

[40] Prathipati P, Alanazi W, Fakhruddin, Jackson DW \& Jackson KE (2015) Role of interstitial angiotensin II and ATP in mediating renal injury induced by recurrent insulin induced hypoglycemia. Annual Research \& Review in Biology 6, 328-36. https://doi.org/10.9734/ARRB/2015/16184.

[41] Pruchniak MP, Araźna M \& Demkow U (2016) Biochemistry of Oxidative Stress. Adv Exp Med Biol. 878, 9-19. https://doi.org/10.1007/5584 2015161.

[42] Quadri S, Prathipati P, Jackson DW \& Jackson KE (2013) Augmentation of heme oxygenase promotes acute angiotensin II induced hypertenion. Clinical and experimental medical sciences 1, 21-43. https://doi.org/10.12988/cems.2013.13003.

[43] Quadri S, Prathipati P, Jackson DW \& Jackson KE (2014) Hemodynamic consequences of recurrent insulin-induced hypoglycemia. Clin Exp Pharmacol Physiol. 41, 81-8. https://doi.org/10.1111/14401681.12183.

[44] Rajagopalan S, Kurz S, Münzel T, Tarpey M, Freeman BA, Griendling KK \& Harrison DG (1996) Angiotensin II mediated hypertension in the rat increases vascular superoxide production via membrane NADH/NADPH oxidase activation. Contribution to alterations of vasomotor tone. J Clin Invest 97, 1916-23. https://doi.org/10.1172/JCI118623.

[45] Ratliff BB, Sekulic M, Rodebaugh J \& Solhaug MJ (2010) Angiotensin II regulates NOS expression in afferent arterioles of the developing porcine kidney. Pediatr Res. 68, 29-34. https://doi.org/10.1203/PDR.0b013e3181e12770.

[46] Razavi Nematollahi L, Kitabchi AE, Stentz FB, Wan JY, Larijani BA, Tehrani MM, Gozashti MH, Omidfar K \& Taheri E (2009) Proinflammatory cytokines in response to insulin-induced hypoglycemic stress in healthy subjects. Metabolism. 58, 443-8. https://doi.org/10.1016/j.metabol.2008.10.018.

[47] Schrader L, Kinzenbaw DA, Johnson AW, Faraci FM \& Didion SP (2007) IL-6 deficiency protects against angiotensin II induced endothelial dysfunction and hypertrophy. Arterioscler Thromb Vasc Biol. 27, 2576-81. https://doi.org/10.1161/ATVBAHA.107.153080.

[48] Shafiee G, Mohajeri-Tehrani M, Pajouhi M \& Larijani B (2012) The importance of hypoglycemia in diabetic patients. J Diabetes Metab Disord. 11, 17. https://doi.org/10.1186/2251-6581-11-17.

[49] Suh SW, Gum ET, Hamby AM, Chan PH \& Swanson RA (2007) Hypoglycemic neuronal death is triggered by glucose reperfusion and activation of neuronal NADPH oxidase. J Clin Invest. 117, 9108. https://doi.org/10.1172/JCI30077. 
[50] The DCCT Research Group (1993) The effect of intensive treatment of diabetes on the development and progression of long term complication in insulin-dependent diabetes mellitus. N Engl J Med. 329 977-86. https://doi.org/10.1056/NEJM199309303291401.

[51] The DCCT Research Group (1997) Hypoglycemia in the Diabetes Control and Complications Trial. Diabetes. 46, 271-86. https://doi.org/10.2337/diab.46.2.271.

[52] Tian XY, Wong WT, Leung FP, Zhang Y, Wang YX, Lee HK, Ng CF, Chen ZY, Yao X, Au CL, Lau CW, Vanhoutte PM, Cooke JP \& Huang Y (2012) Oxidative stress-dependent cyclooxygenase-2-derived prostaglandin F2 $\alpha$ impairs endothelial function in renovascular hypertensive rats. Antioxid. Redox Signaling 16, 363-73. https://doi.org/10.1089/ars.2010.3874.

[53] Toda N, Ayajiki K \& Okamura T (2007) Interaction of endothelial nitric oxide and angiotensin in the circulation. Pharmacol Rev. 59, 54-87. https://doi.org/10.1124/pr.59.1.2.

[54] Touyz RM \& Schiffrin EL (1999) Ang II-stimulated superoxide production is mediated via phospholipase D in human vascular smooth muscle cells. Hypertension. 34, 976-82. https://doi.org/10.1161/01.HYP.34.4.976.

[55] Van der Giet M, Erinola M, Zidek W \& Tepel M (2002) Captopril and quinapril reduce reactive oxygen species. Eur J Clin Invest. 32, 732-7. https://doi.org/10.1046/j.1365-2362.2002.01064.X.

[56] Wang F, Lu X, Peng K, Du Y, Zhou SF, Zhang A, Yang T (2014) Prostaglandin E-prostanoid4 receptor mediates angiotensin II-induced (pro) renin receptor expression in the rat renal medulla. Hypertension. 64, 369-77. https://doi.org/10.1161/HYPERTENSIONAHA.114.03654.

[57] Wei Y, Sowers JR, Nistala R, Gong H, Uptergrove GM, Clark SE Morris EM, Szary N, Manrique C \& Stump CS (2006) Angiotensin II-induced NADPH oxidase activation impairs insulin signaling in skeletal muscle cells. J Biol Chem. 281, 35137-46. https://doi.org/10.1074/jbc.M601320200.

[58] Wong WT, Tian XY, Chen Y, Leung FP, Liu L, Lee HK, Ng CF, Xu A, Yao X, Vanhoutte PM, Tipoe GL \& Huang Y (2010) Bone morphogenic protein-4 impairs endothelial function through oxidative stress-dependent cyclooxygenase-2 upregulation: implications on hypertension. Circ. Res. 107, 984-91. https://doi.org/10.1161/CIRCRESAHA.110.222794.

[59] Wright RJ \& Frier BM (2008) vascular disease and diabetes: is hypoglycaemia an aggravating factor? Diabetes Metab Res Rev. 24, 353-63. https://doi.org/10.1002/dmrr.865.

[60] Wu R, Laplante MA \& de Champlain J (2005) Cyclooxygenase-2 inhibitors attenuate angiotensin II-induced oxidative stress, hypertension, and cardiac hypertrophy in rats. Hypertension. 45, 1139-44. https://doi.org/10.1161/01.HYP.0000164572.92049.29.

[61] Yang H, Jin X, Kei Lam CW \& Yan SK (2011) Oxidative stress and diabetes mellitus. Clin Chem Lab Med. 49, 1773-82. https://doi.org/10.1515/cclm.2011.250.

[62] Yang T (2015) Crosstalk between (Pro) renin receptor and COX-2 in the renal medulla during angiotensin II-induced hypertension. Curr Opin Pharmacol. 21, 89-94. https://doi.org/10.1016/j.coph.2014.12.011.

[63] Zhang C, Hein TW, Wang W \& Kuo L (2003) Divergent roles of angiotensin II AT1 and AT2 receptors in modulating coronary microvascular function. Circ Res. 92, 322-9. https://doi.org/10.1161/01.RES.0000056759.53828.2C. 